\title{
The effect of the incident relative phase on the four-wave mixing field and the electromagnetically induced transparency
}

\author{
Yueping Niu, Shangqing Gong \\ Key Laboratory for High Intensity Optics, \\ Shanghai Institute of Optics and Fine Mechanics, Shanghai 201800, P.R.China
}

\begin{abstract}
In a $\Lambda$-type system employing a two-photon pump field, a four-wave mixing field can be generated simultaneously and hence a closed-loop system forms. We study theoretically on the effect of relative phase between the two incident fields on the generated four-wave mixing field and the electromagnetically induced transparency. It is found that the phase of the generated four-wave mixing field is a sum of the incident relative phase and a fixed phase which is irrelative to the incident relative phase. Hence the total phase of the closed-loop system is independent of the incident relative phase. As a result, the incident relative phase has no effect on electromagnetically induced transparency, which is different from the case of a $\Lambda$-type loop system closed by a third incident field.
\end{abstract}

PACS numbers: 42.50.Gy, 32.80.Qk, 42.50.Hz. 


\section{INTRODUCTION}

The influence of three laser fields of which two often jointly couple to one dipole forbidden transition of a three-level atom makes the generation and amplification of a fourth usually high-frequency field possible. In this aspect, Harris et al. [1] and Petch et al. [2] have studied the generation of four-wave mixing (FWM) theoretically in detail. Dorman and Marangos [3,4] have realized high efficiency of generating four-wave mixing field in krypton atom experimentally. Later, the effect of four-wave mixing on electromagnetically induced transparency (EIT) has also been studied and the asymmetry of EIT profile been demonstrated $[5,6]$.

The effect of electromagnetically induced transparency (EIT) [7] has been the focus of a large number of theoretical and experimental studies in the past few decades. In a simple $\Lambda$-type system, which interacts with two incident fields, the relative phase between the two incident fields will not influence its properties. In a closed-loop $\Lambda$-type three level system, however, the relative phase among the three incident fields not only influences the temporary evolution of the system [8], but also determines the values of the stationary populations in the system [9]. Kosachiov et al. stressed that only at values of general relative phase divisible by $\pi$, did EIT in the system take place. At any other values, the excited level was populated. Furthermore, with increasing of intensity of the applied fields, the $\Lambda$-type closed-loop system became more and more sensitive to the change of the incident relative phase. In a double- $\Lambda$ scheme, Korsunsky et al. have studied the phase-dependent nonlinear optics [10]. They found that at resonance or near-resonance excitation of atoms, particular relations for the field phases, amplitudes, and frequencies ensured the coherent population trapping state. Experimentally, phase-sensitive population dynamics was observed in three$[11,12]$ and four-level $[13,14]$ closed-loop systems.

In the present paper, we investigate the phase of the generated FWM field and the effect of the incident relative phase on EIT in a $\Lambda$-type three level system employing a two-photon pump field [3-6]. The FWM field generates simultaneously and gains a phase of $\theta+\phi$. Because the ground and the lower excited states are closed by the generated FWM field, the total phase of this system becomes $\phi$ and is independent of the incident relative phase $\theta$. Consequently, the incident relative phase does not influence the property of the closed-loop system. 


\section{THE MODEL AND THE GENERATED FOUR-WAVE MIXING FIELD}

We consider an atomic system consisting of a ground state $|c\rangle$, a lower excited state $|b\rangle$ and an upper excited state $|a\rangle$ as shown in Fig.1. Since not all three transitions can be dipole allowed, we here assume the $|a\rangle-|c\rangle$ transition to be of two-photon nature and so that $\omega_{p}$ is selected to be the two-photon pump field. The four-wave mixing field is around the dipole allowed transition $|b\rangle-|c\rangle$ at frequency $\omega_{f}$. The Hamiltonian $H$ in the interaction picture describing the interaction of this three level atom with three laser fields with frequencies $\omega_{d}$, $\omega_{p}$ and $\omega_{f}$ can be cast in the form

$$
H(t)=\left(\begin{array}{ccc}
\Delta_{p} & \Omega_{d} & \Omega_{p} \\
\Omega_{d}^{*} & \Delta_{f} & \Omega_{f} \\
\Omega_{p}^{*} & \Omega_{f}^{*} & 0
\end{array}\right),
$$

where $\Omega_{i}(i=p, d, f)$ refers to the Rabi frequency corresponding to $\omega_{i}(i=p, d, f)$. We assume that $\Omega_{d}=\Omega_{d 0} \exp (-i \theta)$ and $\Omega_{d}^{*}=\Omega_{d 0} \exp (i \theta)$ while $\Omega_{p}^{*}=\Omega_{p} . \quad \theta$ represents the relative

phase which is determined by the phases of the two incident fields and the corresponding transitions. $\Delta_{i}$ corresponds to the detunings given by

$$
\Delta_{p}=\omega_{a c}-\omega_{2 p}, \Delta_{d}=\omega_{a b}-\omega_{d}, \Delta_{f}=\omega_{b c}-\omega_{f}
$$

Here, energy conservation $\Delta_{p}=\Delta_{d}+\Delta_{f}$ has been assumed so that the above Hamiltonian becomes time-independent. Thus, the system Liouville equation for the density matrix element can be written as

$$
\dot{\rho}(t)=-i[H(t), \rho]+\Lambda \rho,
$$

where $\rho$ is the density matrix in the interaction picture and $\Lambda \rho$ represents the irreversible decay part in the system. $\Lambda$ is a phenomenologically added decay terms corresponding to all the incoherent processes. According to steady state solution of Eq.(3), we can obtain the density matrix element $\rho_{b c}$. Expanding it in power of $\Omega_{f}$, we get

$$
\rho_{b c}=A+\left(B_{1} \Omega_{f}+B_{2} \Omega_{f}^{*}\right)+C\left|\Omega_{f}\right|^{2}+\ldots
$$

It is known that the response of a medium to an electric field is governed by its polarization. Consider the coherent radiation field generated by the four-wave mixing process, the polarization can be written as:

$$
P\left(\omega_{f}\right)=N\left(\mu_{b c} \rho_{c b}+\mu_{c b} \rho_{b c}\right)=2 N \mu_{c b} \rho_{b c}
$$


where $N$ denotes the number density of atom with dipole moment $\mu_{c b}$. Because of

$$
P_{l}\left(\omega_{f}\right)=\varepsilon_{0} \chi^{1} E_{f}, \operatorname{and} P_{n l}\left(\omega_{f}\right)=\varepsilon_{0} \chi^{3} E_{p}^{2} E_{d}^{*}
$$

we can get the expression of the FWM field according to the treated methods reported by literature [2]:

$$
E_{f}=\frac{\varepsilon_{0} \chi^{3} E_{p}^{2} E_{d}^{*}}{\chi^{1}}\left[\exp \left(\frac{i \omega_{f} \chi^{1} z}{2 c}\right)-1\right]
$$

Here, phase-match condition is used.

\section{THE EFFECT OF THE INCIDENT RELATIVE PHASE ON EIT}

Solving the equation of motion of the density matrix elements in steady state, we obtain the element $\rho_{b c}$. Though it is complicated, we express it as a power expansion of the Rabi frequency $\Omega_{f}$, just as shown in Eq.(4). Through simple calculation, the relations between the expanding parameters and the incident relative phase are achieved. A is proportional to $\exp (i \theta), B_{2}$ is proportional to $\exp (2 i \theta)$ while $B_{1}$ is independent of the relative phase $\theta$.

Then, we display the quantity $\Omega_{f}$ as a function of the expanding parameters $A, B_{1}$ and $B_{2}$ :

$$
\Omega_{f}=\frac{A B_{1}^{*}-A^{*} B_{2}}{\left(B_{1} B_{1}^{*}-B_{2} B_{2}^{*}\right)} .
$$

It is easy to point out that the Rabi frequency of the FWM field is proportional to $\exp (i \theta)$. Furthermore, because the expanding parameters are complex, we can re-write it as $(a+b i) \exp (i \theta)$. Hence, for the generated FWM field, the corresponding phase is $\theta+\phi . \phi$ is equal to $\arctan (b / a)$, which is determined by the detunings, the Rabi frequencies of the two incident fields and the decaying rates. As the three quantities are determined, $\phi$ becomes a fixed value and is completely independent of the incident relative phase.

In the following, we focus on the effect of the relative phase between the two incident fields on EIT. In this paper, we assume that the relative phase $\theta$ is added to the coupling field, i.e., $\Omega_{d}=\Omega_{d 0} \exp (-i \theta)$. At the same time, the generated FWM field can be expressed as $\Omega_{f}=\Omega_{f 0} \exp [-i(\theta+\phi)]$. Accordingly, the total phase of the closed-loop system is $\phi$. As a result, the incident relative phase has no effect on the population of the upper level $|a\rangle$, i.e. EIT. We show this property in Fig. 2. This phenomenon is completely different from that

of the $\Lambda$-type scheme closed by a third incident field. Just as shown by Kosachiov et al., a radio-frequency field closed their system and its EIT depended on the incident relative phase 
crucially. Only at values of general relative phase divisible by $\pi$, did EIT in the system take place. This is the greatest difference between the two loop schemes closed by an incident field and by a generated field respectively.

In the following, we consider the case of multi-photon resonance. Analytic expressions for $\Omega_{f}$ and $\rho_{a a}$ could be presented concisely. First of all, we discover the FWM field for the stationary case:

$$
\Omega_{f}=\frac{\Omega_{d} \Omega_{p}\left(\Omega_{d}^{2}+\Omega_{p}^{2}\right)}{\gamma\left(\Omega_{d}^{2}-\Omega_{p}^{2}\right)} e^{i(\theta-\pi / 2)} .
$$

It is clear that Eq. (9) fully shows the phase of the generated FWM field. If the relative phase between the two incident fields is $\theta$, the generated FWM field will gain a phase of $\theta-\pi / 2$. Hence, even if the incident relative phase equals to zero, the generated FWM field still achieves a phase of $-\pi / 2$. Solving Eq. (3) under stationary condition, we could obtain the population distribution of the upper excited level $|a\rangle$ :

$$
\rho_{a a}=\frac{2 \gamma^{2} \Omega_{d}^{2} \Omega_{p}^{2}\left(\Omega_{d}^{2}-\Omega_{p}^{2}\right)^{2}}{\gamma^{4} \Omega_{p}^{6}-\Omega_{d}^{4} \Omega_{p}^{2}\left(\gamma^{4}+4 \gamma^{2} \Omega_{p}^{2}-4 \Omega_{p}^{4}\right)+\Omega_{d}^{6}\left(\gamma^{4}+2 \gamma^{2} \Omega_{p}^{2}+4 \Omega_{p}^{4}\right)+\Omega_{d}^{2} \Omega_{p}^{4}\left(2 \gamma^{2} \Omega_{p}^{2}-\gamma^{4}\right)} .
$$

Apparently, now the population of the level $|a\rangle$ is independent of the incident relative

phase $\theta$. In particular, when $\Omega_{d}=\Omega_{p}$, the upper level is unpopulated and EIT appears. Therefore, it is the relative amplitude of the incident fields but not the incident relative phase that control the EIT. Kosachiov et al. has studied the $\Lambda$-type three level system closed by a radio-frequency field and found that the population of the upper level $|a\rangle$ depends not only on the intensity of the fields but also on the relative field phase. By changing the incident relative phase correspondingly, one can both destroy and restore again the EIT. Comparatively, we can say that the response of the loop $\Lambda$-type system closed by the generated FWM field is completely different from the case closed by an incident radiofrequency field.

\section{CONCLUSIONS}

We investigated the phase of the generated four-wave mixing field and the effect of the relative phase between the incident fields on the EIT in a $\Lambda$-type three level system employing a two-photon pump field. Because the phase of the simultaneously generated four-wave mixing field is a sum of the incident relative phase and a fixed phase, the total phase of the 
closed loop system is independent of the incident relative phase. Therefore, it was found that the occurrence of EIT did not lie on the incident relative phase but on the relative amplitude of the two incident fields. This property was different from the ordinary case closed by a third incident field. In general, the behavior of the system was very different in the case of loop interactions closed by a generated FWM field.

\section{Acknowledgement}

This work is supported by the National Natural Sciences Foundation of China (Grant No. 10234030) and the Natural Science Foundation of Shanghai (Grant No. 03ZR14102). 
[1] S. E Harris, J. E Field, A. Imamoglu, Phys. Rev. Lett. 64, 1107(1990).

[2] J.C Petch, C.H Keitel, P.L Knight and J.P Marangos, Phys. Rev. A 53, 543(1996).

[3] C. Dorman, J. P Marangos, Phys. Rev. A 58, 4121(1998).

[4] C. Dorman, I. Kucukkara, J.P Marangos, Opt. Commun. 180, 263(2000).

[5] Y.F Li, Y.P Niu, Opt. Commun. 231, 243(2004).

[6] Y.F Li, Y.P Niu, J. F Sun, Phys. Lett. A 301, 195(2002).

[7] S.E.Harris, Phys.Today 50(7), 36(1997).

[8] Buckle S J, et al., Opt. Acta 33, 1129(1986).

[9] D V Kosachiov, B G Matisov and Yu V Rozhdestvensky, J. Phys. B 25, 2473(1992).

[10] E.A Korsunsky, D. V Kosachiov, Phys. Rev. A 60, 4996(1999).

[11] M.S. Shahriar and P.R. Hemmer, Phys. Rev. Lett. 65, 1865(1990).

[12] K. Yamamoto, K. Ichimura, and N. Gemma, Phys. Rev. A 58, 2460(1998).

[13] W. Maichen, F. Renzoni, I. Mazets, E. Korsunsky, and L. Windholz, Phys. Rev. A 53, 3444 (1996).

[14] N.Ph Georgiades, E.S Polzik, and H.J Kimble, Opt. Lett. 21, 1688(1996).

Fig. 1 Loop $\Lambda$-type three level system closed by the generated four-wave mixing field.

Fig. 2 Population of the upper level $|a\rangle$ (EIT) as functions of the detuning $\Delta_{p}$ and the relative phase $\theta$. Parameters are $\Omega_{d}=\Omega_{p}=0.2$, and $\gamma=0.5$. 


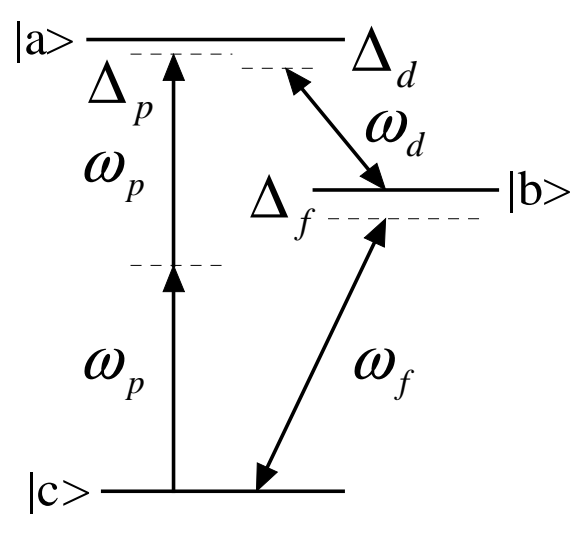

Fig. 1 Niu et al. 


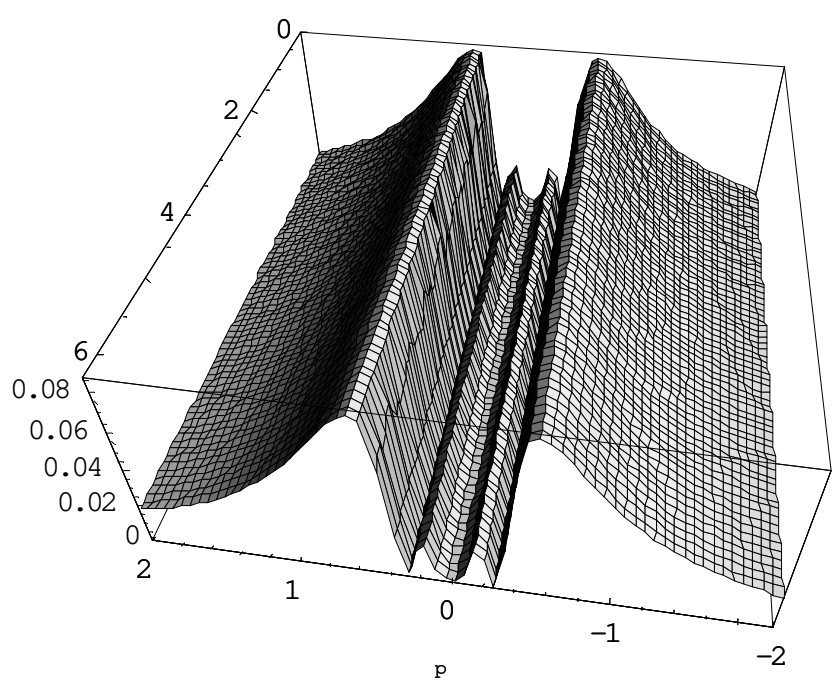

Fig. 2 Niu et al. 